\title{
ARrió $\mid$ Evolução da COVID-19 em Santa Catarina: decretos estaduais e indicadores epidemiológicos até agosto de 2020*
}

doi: $10.1590 /$ S1679-49742021000400025

\section{Evolution of COVID-19 in Santa Catarina, Brazil: state decrees and epidemiological indicators up until August 2020 \\ Evolución de la COVID-19 en Santa Catarina, Brasil: decretos estatales e indicadores epidemiológicos hasta agosto de 2020}

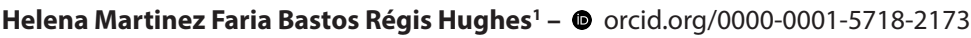 \\ Raquel Alencastro Veiga Domingues Carneiro' ${ }^{1}$ - (1) orcid.org/0000-0002-0770-9771 \\ Danúbia Hillesheim ${ }^{2}$ - (1) orcid.org/0000-0003-0600-4072 \\ Ana Luiza Curi Hallal ${ }^{2}$ - (1) orcid.org/0000-0003-4761-0001 \\ 'Universidade Federal de Santa Catarina, Curso de Graduação em Medicina, Florianópolis, SC, Brasil \\ ${ }^{2}$ Universidade Federal de Santa Catarina, Programa de Pós-Graduação em Saúde Coletiva, Florianópolis, SC, Brasil
}

\section{Resumo}

Objetivo: Descrever a evolução dos decretos e indicadores relacionados à COVID-19 em Santa Catarina, Brasil, até agosto de 2020. Métodos: Estudo ecológico que analisou indicadores epidemiológicos e decretos estaduais sobre o distanciamento social. Os decretos foram agrupados em medidas de restrição, manutenção ou flexibilização. Mortalidade, incidência e transmissibilidade constituíram os indicadores. Resultados: Foram registrados 179.443 casos e 2.183 óbitos no período selecionado. A taxa de incidência passou de 20,4 casos a cada 100 mil habitantes no mês de março para 642,2/100 mil hab. em agosto. Foram emitidos 15 decretos estaduais. Em agosto, quando se observou a maior taxa de mortalidade (13,1/100 mil hab.), verificou-se que todas as categorias, à exceção de uma, haviam sido flexibilizadas. Conclusão: 0s decretos que flexibilizaram as medidas de distanciamento social foram emitidos precocemente, em desacordo com o contexto epidemiológico no estado.

Palavras-chave: Infecções por Coronavírus; COVID-19; Mortalidade; Decretos; Legislação; Epidemiologia Descritiva.

\footnotetext{
*Artigo derivado de monografia de conclusão de curso intitulada 'Evolução da COVID-19 no sul do Brasil: decretos e indicadores no estado de Santa Catarina', defendida por Helena Martinez Faria Bastos Régis Hughes junto ao Curso de Graduação em Medicina da Universidade Federal de Santa Catarina, em 2021.
} 


\section{Introdução}

0 primeiro caso de COVID-19 no Brasil foi confirmado em fevereiro de 2020 e o primeiro óbito em março do mesmo ano. ${ }^{1}$ Em janeiro de 2021, o Brasil ocupava a terceira posição entre os países com maior número de casos confirmados de COVID-19 e a segunda posição em número de óbitos, ao registrar 8.638.249 casos e 212.831 mortes causadas pela doença. ${ }^{1}$ Resultados de um estudo que analisou, retrospectivamente, todos os indivíduos adultos hospitalizados entre as semanas epidemiológicas 8 e 33 de 2020, apontam elevada mortalidade hospitalar por COVID-19 no país, mesmo entre aqueles com menos de 60 anos de idade, agravada pelas disparidades regionais presentes no sistema público de saúde. $^{2}$

\section{Em Florianópolis, capital de Santa Catarina, um estudo apontou divergência entre as medidas de distanciamento e os indicadores epidemiológicos no momento da decisão política.}

A Organização Mundial da Saúde (OMS) destaca que as políticas públicas e sociais têm papel fundamental no sentido de limitar a transmissão do novo coronavírus e diminuir as mortes por COVID-19. ${ }^{3}$ A OMS destaca, ainda, a necessidade de monitoramento regular dos indicadores e de ajuste contínuo das medidas de contenção do vírus SARS-CoV-2, incluindo aquelas de distanciamento social, no nível de transmissão de cada país e região. 0s indicadores de saúde, juntamente com a capacidade de resposta do sistema de saúde, devem ser a base para a adequação e avaliação do impacto, bem como guia para mudanças futuras. ${ }^{3,4}$ As medidas de distanciamento social, como fechamento de escolas e comércio, restrições de viagens, cancelamento de eventos em massa, proibição de aglomerações, entre outras, fazem parte das medidas não farmacológicas recomendadas aos países para 0 controle da pandemia. ${ }^{5-7}$

No Brasil, o caráter emergencial da pandemia demandava uma atuação rápida, cooperativa e solidária das esferas governamentais. No entanto, entende-se que o Poder Executivo Federal subestimou a pandemia e seus efeitos, com sistemáticas tentativas de obstrução às normas promovidas por outros atores, em particular 0 Congresso Nacional e os governos locais. ${ }^{89} \mathrm{Com}$ a regulação da Lei Federal $n^{0} 13.979$, de 6 de fevereiro de 2020, que, entre outros conteúdos, autorizou a promoção de medidas emergenciais de distanciamento social por meio de decretos, governadores e prefeitos tornaram-se protagonistas na produção de atos normativos relacionados à pandemia de COVID-19 em seus territórios. ${ }^{10}$

Em Santa Catarina, o segundo estado mais ao sul do país, de acordo com os dados oficiais, houve 549.579 casos confirmados e 5.988 óbitos causados pela COVID-19 até janeiro de 2021. ${ }^{11}$ Quando comparado aos demais estados brasileiros, em novembro de 2020, Santa Catarina destacou-se em número de novos casos. ${ }^{12}$ Entre março e novembro de 2020, diversos decretos regularam medidas públicas de prevenção e controle da COVID-19, incluindo a promoção de distanciamento social. Um estudo realizado no município de Florianópolis, capital do estado, analisou a relação entre o potencial de propagação do SARS-CoV-2 e as tomadas de decisão do governo municipal quanto ao distanciamento social, até 14 de julho de 2020. 0s resultados apontaram divergência entre as medidas de distanciamento e os indicadores epidemiológicos no momento da decisão política. ${ }^{13}$

Considerando-se 0 impacto da pandemia da COVID-19 no Brasil, as importantes diferenças regionais no país e o elevado número de casos e óbitos em Santa Catarina, faz-se necessário avaliar, com maior grau de detalhamento, o contexto epidemiológico do estado, no qual houve implementação, manutenção e/ou flexibilização de medidas de distanciamento social. 0 presente estudo teve como objetivo descrever a evolução dos decretos e indicadores relacionados à COVID-19 no estado de Santa Catarina, Brasil, até o mês de agosto de 2020 .

\section{Métodos}

Trata-se de um estudo ecológico, realizado em Santa Catarina. 0 período estudado constituiu-se da

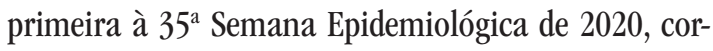
respondendo ao intervalo entre os dias 29 de dezembro de 2019 e 29 de agosto de 2020. Foram analisados os casos e óbitos confirmados para COVID-19 em residentes no estado. Foram considerados casos ou óbitos 
aqueles que possuíam confirmação laboratorial para SARS-CoV-2, mediante teste por biologia molecular, teste imunológico ou pesquisa de antígeno, independentemente dos sinais ou sintomas apresentados. ${ }^{14}$

A fonte dos dados utilizada para a consulta de casos e óbitos foi o portal de dados abertos do estado de Santa Catarina, ${ }^{15}$ consultado no dia 11 de setembro de 2020. As informações sobre a população residente foram obtidas no sítio eletrônico do Departamento de Informática do Sistema Único de Saúde do Brasil, considerando-se a projeção da população de Santa Catarina estimada para 2020. As estimativas da taxa de transmissibilidade (Rt) são oriundas do painel da Rede CoVida ${ }^{16}$ coletadas no primeiro dia de cada mês. 0 Rt, uma métrica matemática que indica quão contagiosa uma doença é, foi calculado por meio da curva dos casos previstos pelo modelo matemático de predição de casos.

As variáveis analisadas, referentes à caracterização do contexto epidemiológico, foram:

a) número de casos novos (segundo a data de início dos sintomas);

b) número de óbitos nas últimas 24 horas (segundo a data do óbito);

c) taxa de mortalidade;

d) taxa de incidência;

e) taxa de transmissibilidade ajustada, Rt; e

f) número de municípios com casos e número de municípios com óbitos.

0s dados foram descritos segundo os meses do calendário. As taxas de incidência e de mortalidade foram obtidas dividindo-se o número de casos e o número de óbitos nas últimas 24 horas, respectivamente, pela população residente e multiplicando-se por 100 mil. 0s dados foram analisados utilizando-se o software Stata versão 14 .

Os decretos consultados no estudo também foram identificados no portal de dados abertos de Santa Catarina, ${ }^{15}$ e extraídos, em seu completo teor, do Diário Oficial do Estado. Foram analisados os decretos estaduais de Santa Catarina que regulam matéria relacionada à Lei $n^{0} 13.979 / 2020$. As variáveis analisadas nos diferentes decretos foram: número do decreto; data de sua publicação; data de sua entrada em vigor; e medida de distanciamento social implementada e sua repercussão sobre a categoria, em relação à normativa anterior. A análise teve por objetivo avaliar medidas de distanciamento social, sendo criadas nove categorias de agrupamento de atividades. Primeiramente, foram selecionadas, durante a análise dos decretos, as atividades relacionadas ao distanciamento social ${ }^{17}$ e cujo funcionamento permitiu maior contato entre indivíduos - possível de ser evitado ou reduzido em caso de limitação da atividade. Para a criação das categorias, utilizaram-se tanto agrupamentos já existentes nos próprios decretos quanto moldes de agrupamentos semelhantes aos de outras literaturas, ${ }^{17,18}$ a saber:

a) ensino presencial - incluídos todos os níveis educacionais, desde o ensino básico até 0 universitário, assim como a educação de jovens e adultos, o ensino técnico e a capacitação pública;

b) comércio de bebidas e alimentos não essenciais - incluídos restaurantes, bares e demais estabelecimentos que fornecem alimentos e bebidas não essenciais;

c) transporte de pessoas - incluídos os transportes aéreo, terrestre ou aquaviário;

d) rede hoteleira - incluídos hotéis, pousadas ou qualquer forma de hospedagem onerosa;

e) comércio de produtos - incluído o comércio de produtos não alimentícios e não essenciais, em geral;

f) comércio de alimentos essenciais - incluídos supermercados, mercados, mercearias, padarias, açougues e peixarias;

g) permanência em praças e locais públicos - incluídos espaços públicos de uso coletivo, como praias, parques e praças;

h) cultos religiosos - incluídos culto de caráter religioso e missas; $\mathrm{e}$

i) aglomeração de pessoas - incluída qualquer regulação sobre a matéria, especificada ou não pelo redator, desde que haja menção literal do termo.

A repercussão dos decretos sobre as categorias de agrupamentos, por sua vez, foi classificada em três categorias:

(i) Restrição

Repercussão provocada por decreto cujo texto apresenta medidas restritivas iniciais para a categoria ou medidas cujos efeitos restritivos superam aqueles em vigor, anteriormente ao decreto. Para fins de superação, considerou-se o impacto da medida e não o prazo de sua vigência. 
(ii) Manutenção

Quando um novo decreto prorroga o prazo de vigência de medidas restritivas de decretos anteriores, com produção de iguais efeitos.

(iii) Flexibilização

Para o caso de um novo decreto cujo impacto do teor restritivo se percebe diminuído, em relação ao decreto anterior; ou decreto que transcorre o prazo de vigência do anterior, sem adicionar novas restrições na categoria.

Alguns indicadores foram apresentados conforme a Matriz de Risco Potencial da COVID-19, divulgada pela Secretaria de Estado da Saúde de Santa Catarina. ${ }^{19}$ A partir da combinação de indicadores, essa matriz classifica as regiões do estado em quatro níveis de risco para a infecção: moderado, alto, grave ou gravíssimo. ${ }^{19}$

Em razão de os dados utilizados serem de domínio público e sem a identificação dos participantes, 0 projeto do estudo foi dispensado de avaliação por um Comitê de Ética em Pesquisa com Seres Humanos.

\section{Resultados}

Foram registrados 179.443 casos - confirmados laboratorialmente - para COVID-19 em residentes no estado de Santa Catarina, e 2.183 óbitos, até o final da Semana Epidemiológica 35 de 2020. 0s meses de julho e agosto registraram o maior número de casos novos e de óbitos no período, respectivamente 87.143 e 947. A taxa de incidência passou de 20,4 casos a cada 100 mil hab. no mês de março para 1.201,6/100 mil hab. em julho e 642,2/100 mil hab. em agosto. No mesmo mês de agosto, observou-se a maior taxa de mortalidade no período analisado: 13,1 óbitos por 100 mil hab. (Tabela 1).

No primeiro dia do mês de abril, uma pessoa infectante produziu, em média, três infecções secundárias $(\mathrm{R} t=3,0)$. Nos meses subsequentes, esse indicador foi de 1,2. No mês de agosto de 2020, todos os 295 municípios do estado possuíam casos notificados da doença, enquanto os óbitos estavam presentes em $69,8 \%$ deles (Tabela 1).

No período estudado, foram emitidos 31 decretos estaduais sobre o SARS-CoV-2 em Santa Catarina: três legislativos e 28 executivos. Entre os 31 decretos observados, 15 tratavam de medidas de restrição, flexibilização ou manutenção do distanciamento social das categorias de agrupamento de atividades analisadas.
No mês de março de 2020 , foram emitidos seis decretos, todos com efeitos restritivos ou de manutenção da restrição. Em abril, foram emitidos quatro decretos, com sete repercussões de flexibilização nas categorias analisadas, a saber: comércio de bebidas e alimentos não essenciais (categoria contemplada em dois decretos com flexibilizações); e rede hoteleira, comércio de produtos, permanência em praças e locais públicos, cultos religiosos e aglomeração de pessoas (cada uma destas categorias contemplada com flexibilização). No mês de junho, foi emitido um decreto com flexibilização das medidas quanto a ensino presencial, transporte de pessoas e aglomeração de pessoas. Nos meses de julho e agosto, foram emitidos três decretos e um decreto, respectivamente, em que as principais características foram, por um lado, a manutenção das flexibilizações de medidas, e por outro lado, sua restrição para os municípios de regiões classificadas como de risco gravíssimo, segundo a Matriz de Risco Potencial da COVID-19, divulgada pela Secretaria de Estado da Saúde. No mês de agosto, praticamente todas as categorias - à exceção de uma - haviam sofrido flexibilizações e não houve decretos restritivos (Figura 1).

A Figura 2 apresenta a evolução temporal do número de casos novos de COVID-19 e dos decretos estaduais relacionados a medidas de distanciamento social em Santa Catarina. No mês de abril, observaram-se os primeiros decretos com flexibilizações do distanciamento social nas categorias analisadas, sendo que, entre os dias 11 de abril e $1^{\circ}$ de junho, foram emitidos os decretos de números 554, 587 e 630, resultando na flexibilização de todas as categorias de agrupamento de atividades analisadas, à exceção da categoria 'comércio de alimentos essenciais'. No período transcorrido entre a publicação dos decretos de números 554 e 630, houve um aumento de $1.805 \%$ no número de novos casos por dia. 0 primeiro decreto publicado após esse período estabelecia restrições de atividades para os municípios de regiões classificadas sob risco gravíssimo, segundo a Matriz de Risco Potencial da COVID-19 definida pelo estado (Figura 2).

A Figura 3 apresenta a evolução temporal do número de óbitos por COVID-19 nas últimas 24 horas, acompanhado da série de decretos estaduais relacionados a medidas de distanciamento social em Santa Catarina. 0s primeiros cinco decretos que restringiram atividades - de números 507, 509, 515, 521 e 525 - foram publicados quando ainda não havia registro de óbitos por COVID-19 no estado. 
Tabela 1 - Indicadores da COVID-19 segundo mês de ocorrência em Santa Catarina, até agosto de 2020

\begin{tabular}{|c|c|c|c|c|c|c|c|}
\hline \multirow[t]{2}{*}{ Mês } & Casos $^{\mathrm{a}}$ & Óbitos & \multirow{2}{*}{$\begin{array}{l}\text { Taxa de incidência } \\
\text { (por } 100 \text { mil hab.) }\end{array}$} & \multirow{2}{*}{$\begin{array}{l}\text { Taxa de mortalidade } \\
\text { (por } 100 \text { mil hab.) }\end{array}$} & \multirow{2}{*}{$\mathbf{R t}^{c}\left(\mathbf{I C}_{95 \%}{ }^{d}\right)$} & \multirow{2}{*}{$\begin{array}{c}\text { Municípios } \\
\text { com casos }\end{array}$} & \multirow{2}{*}{$\begin{array}{c}\text { Municípios } \\
\text { com óbitos }\end{array}$} \\
\hline & $\mathbf{n}$ & $\mathbf{n}$ & & & & & \\
\hline Fevereiro & 30 & - & 0,4 & - & & $17(5,7)$ & - \\
\hline Março & 1.483 & 5 & 20,4 & 0,1 & & $116(39,3)$ & $5(1,6)$ \\
\hline Abril & 2.936 & 49 & 40,5 & 0,7 & $3,0(0,7 ; 7,8)$ & $180(61,0)$ & $31(10,5)$ \\
\hline Maio & 9.698 & 95 & 133,7 & 1,3 & $1,2(0,9 ; 1,5)$ & $244(82,7)$ & $57(19,3)$ \\
\hline Junho & 31.576 & 229 & 435,4 & 3,2 & $1,2(0,9 ; 1,4)$ & $281(95,2)$ & $89(30,1)$ \\
\hline Julho & 87.143 & 858 & $1.201,6$ & 11,8 & $1,2(1,0 ; 1,3)$ & $295(100,0)$ & $162(54,9)$ \\
\hline Agosto & 46.577 & 947 & 642,2 & 13,1 & $1,2(1,0 ; 1,3)$ & $295(100,0)$ & $206(69,8)$ \\
\hline Total & 179.443 & 2.183 & $2.474,2$ & 30,1 & & $295(100,0)$ & $295(100,0)$ \\
\hline
\end{tabular}

a) Número de casos novos a cada mês; b) Número de óbitos registrados a cada mês; c) Rt: taxa de transmissibilidade ajustada, referente ao primeiro dia do mês; d) $I_{95 \%}$ : intervalo de confiança de $95 \%$.

Nos meses de maio, junho, julho e agosto de 2020, em contraste com o crescimento do número de óbitos, não foram emitidos decretos restritivos - à exceção do Decreto Estadual n ${ }^{0} 724$, de 17 de julho de 2020, referente apenas aos municípios de regiões classificadas sob risco gravíssimo, segundo a Matriz de Risco Potencial da COVID-19, da Secretaria de Estado da Saúde de Santa Catarina (Figura 3).

\section{Discussão}

Da primeira à 35ª Semana Epidemiológica de 2020, houve crescimento das taxas de incidência e mortalidade por COVID-19 no estado de Santa Catarina. No período, o estado emitiu 31 decretos estaduais regulando matérias relacionadas ao SARS-CoV-2 e quase metade deles repercutiu sobre o distanciamento social nas categorias analisadas. No mês de agosto, quando se observou a maior taxa de mortalidade pela doença no estado, verificou-se flexibilização para todas as categorias analisadas, com exceção de uma.

0 crescimento das taxas de mortalidade e incidência no estado de Santa Catarina ocorreu em diferentes proporções. Comparado a outros estados brasileiros, Santa Catarina se manteve entre as menores mortalidades por SARS-CoV-2 no país, durante todo o período estudado.$^{20} \mathrm{Um}$ estudo descreveu a evolução da COVID-19 no Brasil até a 20 Semana Epidemiológica e apontou para Santa Catarina a sexta menor taxa de mortalidade pela doença no país e nona menor taxa de incidência. ${ }^{21}$ Até o final da $35^{\text {a }}$ Semana Epidemiológica, o estado ocupava a oitava posição quanto ao número de casos confirmados de COVID-19 ${ }^{22}$ e respondia pela $11^{a}$ maior taxa de incidência da doença no país. ${ }^{20} \mathrm{~A}$ Rt ajustada iniciou-se em 3,0 no mês de abril, decresceu para 1,2 em maio de 2020 e assim se manteve, constante, até o final do período. Tais números são consistentes com as taxas nacionais encontradas em estudo sul-americano, que aponta Rt inicial, após 14 dias da primeira infecção no território nacional, de 3,4; e Rt de 1,2 após 90 dias. $^{23}$

Um projeto brasileiro, de 'Mapeamento e análise das normas jurídicas de resposta à COVID-19 no Brasil' entre janeiro e dezembro de 2020, revelou Santa Catarina como um dos estados com maior produção de normas relacionadas ao coronavírus até abril de $2020 .{ }^{24}$ Contudo, o maior número de normativas não necessariamente refletiu uma coerência com o contexto epidemiológico da COVID-19 no estado.

No período estudado, nove dos 15 decretos estaduais destinados à regulação da matéria do distanciamento social das categorias elencadas impactaram em restrição ou flexibilização das atividades previstas pela categoria. 0s primeiros cinco decretos precederam o primeiro óbito por COVID-19 no estado e possuíam caráter restritivo. 0 primeiro decreto entrou em vigor na $12^{a}$ Semana Epidemiológica de 2020 e, em apenas uma semana, todas as categorias já haviam sofrido restrições. Um estudo brasileiro sobre 0 tema, ${ }^{18}$ dedicado à caracterização das medidas de distanciamento social iniciais, implementadas pelos estados, concluiu que, na maioria deles, as medidas de distanciamento social foram implementadas de maneira precoce, considerando-se a fase da curva de crescimento exponencial de casos e de óbitos pela COVID-19. 


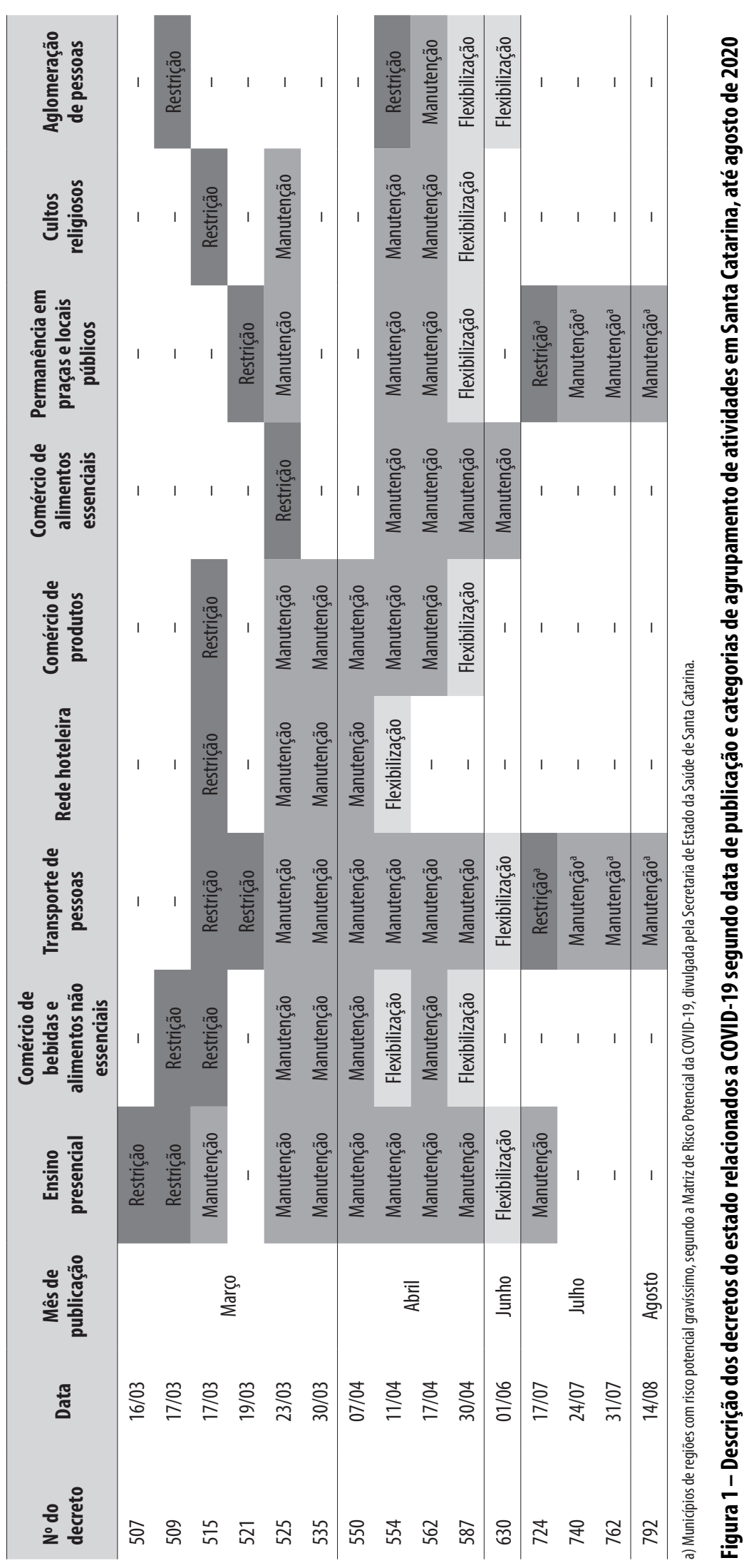




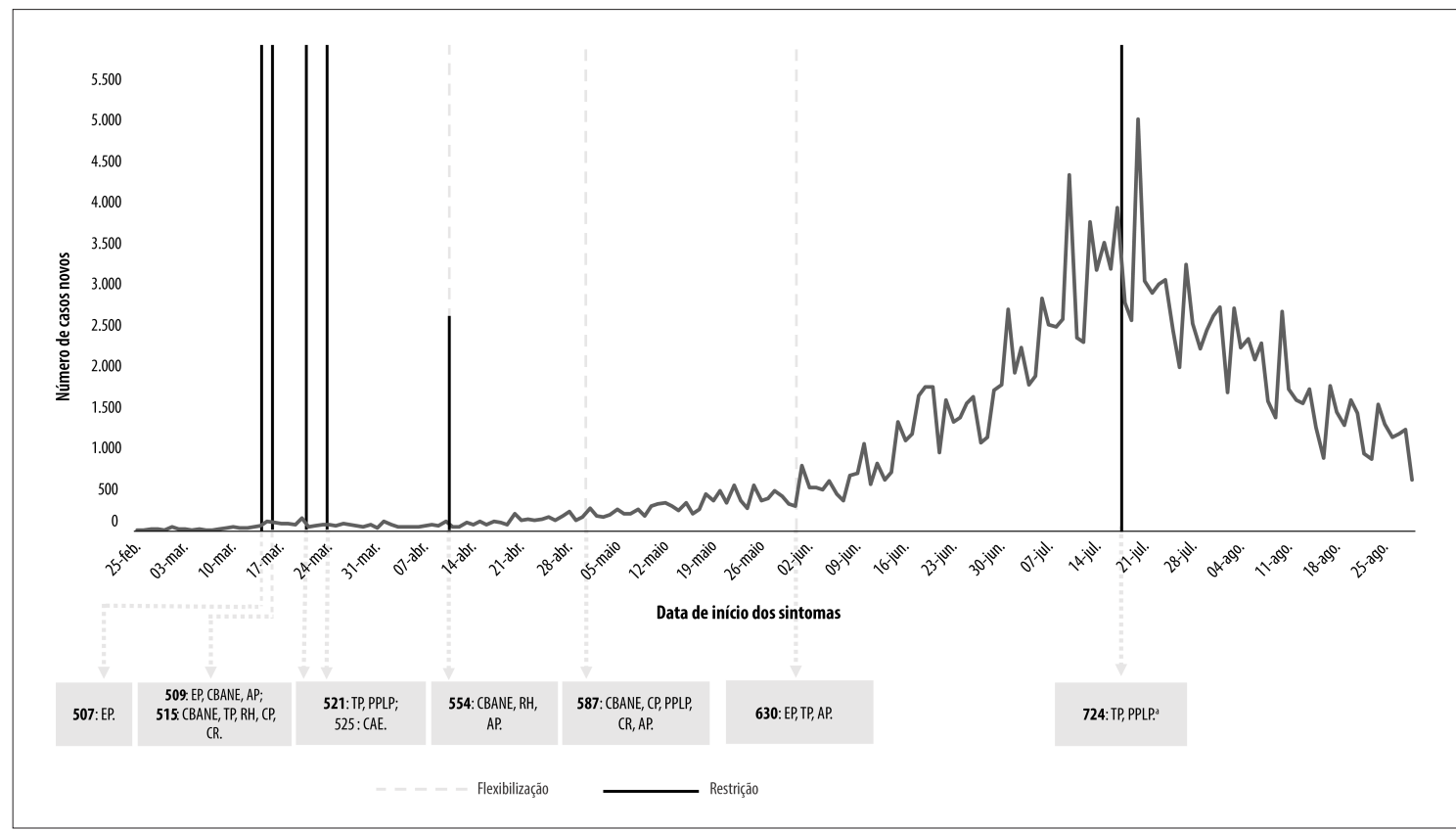

a) Municípios de regiões com potencial gravíssimo, segundo a Matriz de Risco Potencial da COVID-19, divulgada pela Secretaria de Estado da Saúde de Santa Catarina.

Legenda: EP: ensino presencial; CBANE: comércio de bebidas e alimentos não essenciais; TP: transporte de pessoas; RH: rede hoteleira; CP: comércio de produtos; CAE: comércio de alimentos essenciais; PPLP: permanência em praças e locais públicos; CR: cultos religiosos; AP: aglomeração de pessoas.

Figura 2 - Evolução temporal do número de casos novos de COVID-19 e dos decretos do estado relacionados à doença, que flexibilizaram ou restringiram as categorias de agrupamento de atividades em Santa Catarina, até agosto de 2020

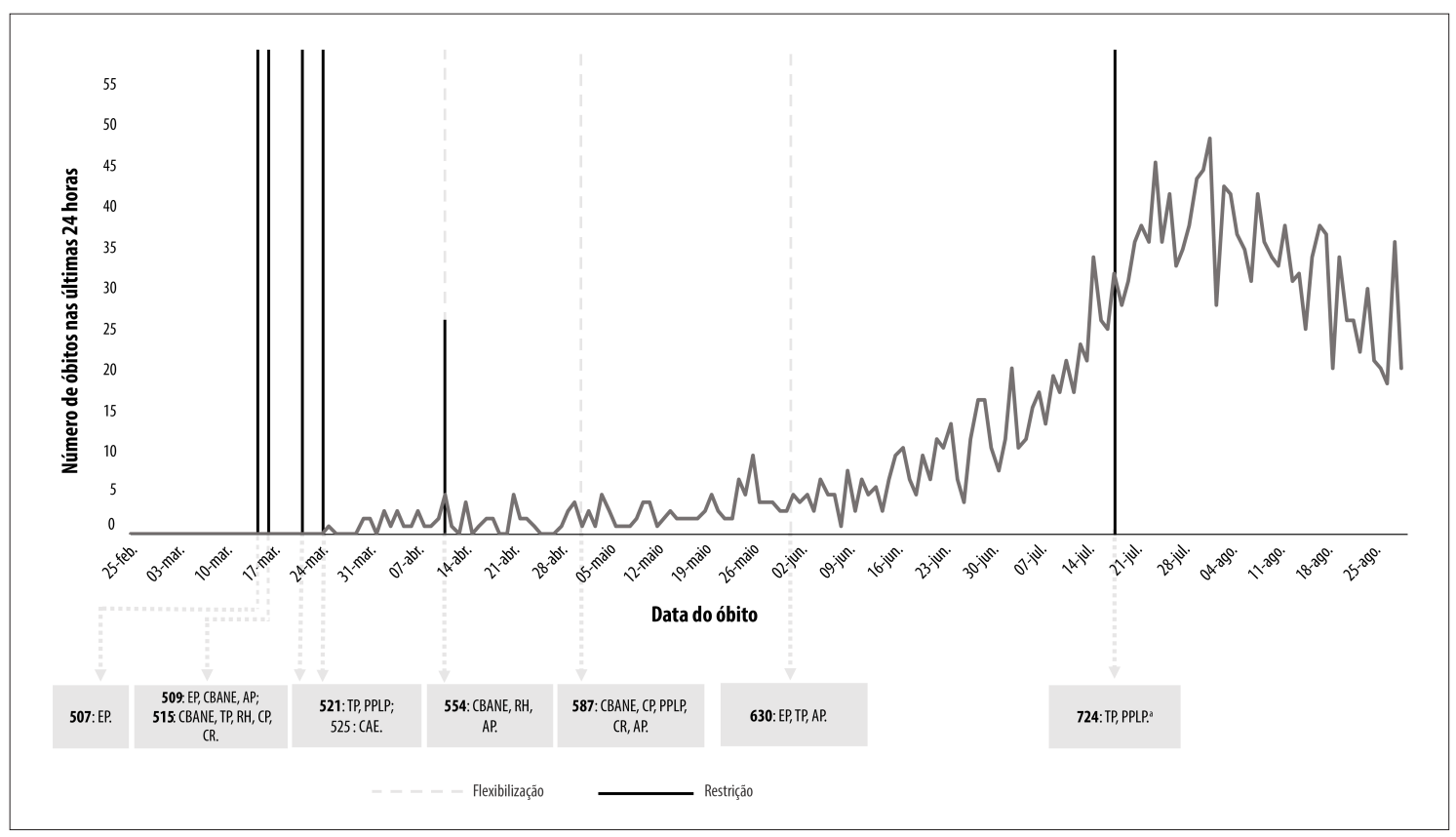

a) Municípios de regiões com potencial gravíssimo, segundo a Matriz de Risco Potencial da COVID-19, divulgada pela Secretaria de Estado da Saúde de Santa Catarina. Legenda: EP: ensino presencial; CBANE: comércio de bebidas e alimentos não essenciais; TP: transporte de pessoas; RH: rede hoteleira; CP: comércio de produtos; CAE: comércio de alimentos essenciais; PPLP: permanência em praças e locais públicos; CR: cultos religiosos; AP: aglomeração de pessoas.

Figura 3 - Evolução temporal do número de óbitos nas últimas 24 horas e dos decretos do estado relacionados à doença, que flexibilizaram ou restringiram as categorias de agrupamento de atividades em Santa Catarina, até agosto de 2020 
0 mesmo estudo classificou o estado de Santa Catarina na categoria 'intermediário', de acordo com o momento epidemiológico em que cada estado implementou as categorias de medidas de distanciamento social segundo 0 número de casos confirmados de COVID-19. ${ }^{18}$ Dessa forma, observou-se que o Governo do Estado de Santa Catarina foi ágil em estabelecer restrições iniciais.

A importância da implementação de medidas de distanciamento social precocemente, para a contenção da COVID-19, foi amplamente discutida na literatura. Lai et al. sugerem, por meio de um modelo matemáti$\mathrm{co}$, que medidas de distanciamento social poderiam ter reduzido o número de casos de SARS-CoV-2 na China em $95 \%$, se aplicadas três semanas antes do período realizado. ${ }^{6}$ Outro estudo, este nos Estados Unidos, ao avaliar os efeitos do distanciamento social na taxa de mortalidade por COVID-19 naquele país, sugere que, passados os sete primeiros dias da implementação de medidas de distanciamento social, o crescimento da taxa de mortalidade decai cerca de $2 \%$ ao dia, fortalecendo a importância de uma antecipada implementação de medidas de distanciamento social. ${ }^{25}$

Em se tratando da obrigatoriedade dessas medidas e seus efeitos, um estudo cujo objetivo foi analisar o impacto de medidas de distanciamento social no Reino Unido, nas taxas de isolamento social e na taxa de transmissibilidade estimada (Ro) da COVID-19, encontrou uma redução de $74 \%$ no contato interpessoal e uma redução de 2,6 para 0,62 na taxa de transmissibilidade estimada. ${ }^{7} \mathrm{E}$ os resultados de um estudo brasileiro, que se propôs a identificar alguns dos potenciais determinantes dos níveis de distanciamento social no país, demonstraram que normas de imposição do distanciamento social têm mais efeitos do que a percepção do número de casos, sugerindo que as pessoas respondem melhor à normas do que à severidade da pandemia. ${ }^{26}$

Conscientes da consolidada importância da elaboração e implementação de normas reguladoras do distanciamento social, governadores de estados brasileiros enfrentaram uma encruzilhada federativa para legislar sobre a matéria. ${ }^{27}$ No período entre as publicações dos decretos 521 e 525, o Governo Federal adotou a Medida Provisória (MP) $n^{0}$ 926, de 20 de março de $2020,{ }^{28}$ que, entre outros conteúdos, alterou a Lei $\mathrm{n}^{\circ}$ 13.979, de 6 de fevereiro de 2020, com o objetivo de limitar a competência de governadores e prefeitos na expedição de decretos, conferindo apenas à União 0 direito de decidir sobre certas restrições. Destaca-se que, naquele momento, a Lei $\mathrm{n}^{0}$ 13.979/2020 vigorava há 14 dias, e todas as 27 Unidades da Federação - incluído o Distrito Federal - já haviam expedido decretos regulando-a. ${ }^{18} \log 0$, a proposta de alteração da MP $n^{0}$ 926/2020 foi alvo de ação direta de inconstitucionalidade (ADI) ${ }^{28}$ No dia 24 de março de 2020 , o Supremo Tribunal Federal deferiu em parte um pedido de liminar proposto na ADI $n^{0}$ 6.341, a fim de reconhecer a competência concorrente entre estados, municípios e União na tomada de providências normativas e administrativas para 0 enfrentamento do coronavírus. ${ }^{28}$ Assim, a dispor da intercorrência, estados e municípios tiveram assegurado seu direito constitucional de legislar sobre saúde pública.

0 momento da expedição de decretos é tão importante quanto seus conteúdos, para a desaceleração do número de casos e óbitos por COVID-19.6,29 Entre os impactos dos decretos emitidos pelo estado de Santa Catarina, da primeira à $35^{\mathrm{a}}$ Semana Epidemiológica, as flexibilizações requerem especial atenção, dado 0 momento de sua implementação. De 11 de abril a $1^{0}$ de junho de 2020, foram expedidos três decretos de flexibilização, concomitantemente ao crescimento de $1.804,8 \%$ no número absoluto de novos casos da doença.

No que concerne ao momento de flexibilização de medidas de distanciamento social, vê-se como prematura a flexibilização das restrições antes do alcance de uma taxa de transmissibilidade (Rt) abaixo de 1,0. ${ }^{30}$ De tal forma, todas as flexibilizações decretadas pelo estado catarinense, inclusive na emissão dos decretos de números 554, 587 e 630, ocorreram de forma precoce, uma vez que o Rt encontrado nesses momentos era de $1,2 .{ }^{16}$ Soma-se a isso o fato de a flexibilização de medidas no estado ter-se dado de forma abrupta, em desacordo com o preconizado na literatura., ${ }^{5,29}$

Novas restrições foram estabelecidas apenas nos quatro últimos decretos emitidos, no âmbito do período analisado; e mesmo assim constituíram-se, tão somente, de restrições parciais. Aplicadas sobre as categorias 'transporte de pessoas' e 'permanência em praças e locais públicos', tais restrições promoveram distanciamento social unicamente nos municípios de regiões consideradas sob risco potencial gravíssimo. Esses decretos foram emitidos entre as semanas epidemiológicas 29 e 33, quando o estado de Santa Catarina enfrentava o pior momento epidemiológico do período 
avaliado, possuía o maior número de novos casos da doença na região Sul e alcançava o quarto maior número de novos casos no Brasil, referentes à $31^{\text {a }}$ Semana Epidemiológica. ${ }^{20}$

Efetivamente, verificou-se a flexibilização total de seis das nove categorias de atividades analisadas de $1^{\circ}$ de junho de 2020 até o final do período estudado, apesar das crescentes taxas de mortalidade e incidência no estado de Santa Catarina. Tais achados são consistentes com os resultados de outros estudos brasileiros, que encontraram inadequações no relaxamento de medidas de distanciamento social frente aos dados epidemiológicos. ${ }^{16}$

Este estudo apresenta potenciais limitações, inerentes ao desenho epidemiológico e às fontes dos dados consultadas. A escolha pela utilização de dados secundários, tanto para identificação dos casos como dos óbitos por COVID-19, submete os resultados (i) à influência da disponibilidade de testes para o diagnóstico laboratorial em Santa Catarina, ao longo do período, (ii) à qualidade do preenchimento das fichas de investigação e (iii) à abrangência dos sistemas de informações em saúde envolvidos na notificação de casos ambulatoriais e hospitalizados, a saber, e-SUS VE e Sistema de Vigilância Epidemiológica da Gripe . Além disso, decretos municipais e outras formas de legislação não foram objeto de análise no presente estudo.

\section{Referências}

1. Johns Hopkins Coronavirus Resource Center. COVID-19 Map [Website]. 2021 [citado em 26 jan 2021]. Disponível em: https://coronavirus.jhu.edu/map.html

2. Ranzani OT, Bastos LSL, Gelli JGM, Marchesi JF, Baião F, Hamacher S, et al. Characterisation of the first 250000 hospital admissions for COVID-19 in Brazil: a retrospective analysis of nationwide data. Lancet Respiratory Medicine. 2021;15:S2213-2600(20)305609. doi: https://doi.org/10.1016/S2213-2600(20)30560-9.

3. World Health Organization. Considerations for implementing and adjusting public health and social measures in the context of COVID-19: interim guidance, 4 November 2020. Geneva: WHO; 2020 [acesso 19 out. 2021]. Disponível em: https://apps.who.int/iris/handle/10665/336374.

4. Viana N. Gestão estadual do SUS: avaliação da transparência. In: Santos A0, Lopes LT, organizadores.
Conclui-se que, no estado de Santa Catarina, houve crescimento do número de casos e óbitos por COVID-19, bem como das taxas de incidência e de mortalidade até a Semana Epidemiológica 35 de 2020, acompanhado de elevação nas taxas de incidência e mortalidade no período estudado. 0s decretos que flexibilizaram as medidas de distanciamento social, recomendadas para conter a transmissão e reduzir a morbidade e mortalidade por COVID-19, foram emitidos precocemente, em desacordo com o contexto epidemiológico da infecção no estado de Santa Catarina.

\section{Contribuição das autoras}

Hillesheim D participou da análise e interpretação dos dados, elaboração do manuscrito e revisão crítica de seu conteúdo. Hughes HMFBR e Carneiro RAVD participaram da análise e interpretação dos dados, concepção do estudo, redação, revisão de literatura e revisão crítica do conteúdo do manuscrito. Hallal ALC participou da concepção do estudo, redação e revisão crítica do conteúdo do manuscrito. Todas as autoras aprovaram a versão final do manuscrito e assumem responsabilidade por todos os seus aspectos, garantindo a precisão e integridade da obra.
Competências e regras [Internet]. Brasília, DF: CONASS; 2021 [acesso 20 out. 2021]. (Coleção covid-19, vol. 3). p. 188-207. Disponível em: https://www.conass. org.br/biblioteca/volume-3-competencias-e-regras/

5. Organização Pan-Americana de Saúde. Medidas não farmacológicas para a redução da disseminação do vírus. Brasília, DF: OPAS; [2020] [acesso 21 jan. 2021]. Disponível em: https://opascovid.campusvirtualsp. org/covid-19/medidas-nao-farmacologicaspara-reducao-da-disseminacao-do-virus

6. Lai S, Ruktanonchai NW, Zhou L, Prosper 0, Luo W, Floyd JR, et al. Effect of non- pharmaceutical interventions to contain covid-19 in China. Nature. 2020;585(7825):410-3. doi: https:// doi.org/10.1038/s41586-020-2293-X.

7. Jarvis CI, Van Zandvoort K, Gimma A, Prem K, Klepac P, CMMID COVID-19 working group, et 
al. Quantifying the impact of physical distance measures on the transmission of covid-19 in the UK. BMC Medicine. 2020;18(1):154. doi: https:// doi.org/10.1186/s12916-020-01597-8.

8. Conectas Direitos Humanos. Mapeamento e análises das normas jurídicas de resposta à covid-19 no Brasil. Bol Direitos Pandemia. 20 jan. 2021 [acesso 26 jan. 2021];(10)1-56. Disponível em: https://www.conectas.org/publicacoes/ download/boletim-direitos-na-pandemia-no-10

9. The Lancet. Covid-19 in Brazil: "So what?". Lancet. 2020 May 9;395(10235):1461. doi: https:// doi.org/10.1016/S0140-6736(20)31095-3.

10. Terrazas FV. Legislação para o enfrentamento da covid-19: leis e atos administrativos. In: Santos A0, Lopes LT, organizadoras. Competências e regras [Internet]. Brasília, DF: CONASS; 2021 [acesso 20 out. 2021]. (Coleção covid-19, vol. 3). p. 248268. Disponível em: https://www.conass.org.br/ biblioteca/volume-3-competencias-e-regras/

11. Governo de Santa Catarina. Coronavírus: boletim epidemiológico [Internet]. [Florianópolis (SC]: Secretaria de o Estado da Administração]; 2021 [acesso 20 jan. 2021]. Disponível em: http://www. coronavirus.sc.gov.br/wp-content/uploads/2021/01/ boletim-epidemiologico-20-01-2021.pdf

12. Ministério da Saúde (BR). Covid-19 no Brasil: painel [Website]. c2020 [acesso 3 nov. 2020. Disponível em: https://susanalitico.saude.gov.br/ extensions/covid-19_html/covid-19_html.html

13. Garcia LP, Traebert J, Boing AC, Santos GFZ, Pedebôs LA, d'Orsi E, et al. 0 potencial de propagação da covid-19 e a tomada de decisão governamental: uma análise retrospectiva em Florianópolis, Brasil. Rev Bras Epidemiol. 2020;23:e200091. doi: http://doi.org/10.1590/1980-549720200091.

14. Diretoria de Vigilância Epidemiológica de Santa Catarina, Secretaria do Estado de Saúde (SC). Manual de orientações da covid-19: vírus SARS-CoV-2 [Internet]. [Florianópolis (SC]: SES; 2020 [acesso 3 nov. 2020]. Disponível em: https://www.saude.sc.gov.br/ coronavirus/arquivos/Manual_23-10-atualizado.pdf

15. Governo do Estado de Santa Catarina (SC).

Portal de dados abertos do estado de Santa

Catarina [Website]. c2016 [acesso 9 nov. 2020].

Disponível em: http://dados.sc.gov.br
16. Rede Covida Ciência Informação e Solidariedade. Painel [Website]. - Rede CoVida [Internet]. 2020 [citado em 26 jan 2021]. Disponível em: https://painel.redecovida.org/brasil

17. Aquino EML, Silveira IH, Pescarini JM, Aquino R, Souza-Filho JA, Rocha AS, et al. Social distancing measures to control the covid-19 pandemic: potential impacts and challenges in Brazil. Cien Saude Colet. 2020;25(suppl 1):2423-46. doi: https://doi. org/10.1590/1413-81232020256.1.10502020.

18. Silva LLS, Lima AFR, Polli DA, Razia PFS, Pavão LFA, Cavalcanti MAFH, et al. Social distancing measures in the fight against covid-19 in Brazil: description and epidemiological analysis by state. 2020 Sep 18;36(9):e00185020. doi: https:// doi.org/10.1590/0102-311x00185020.

19. Governo de Estado de Santa Catarina, Gestão da Saúde. Coronavírus: avaliação do risco potencial para covid-19 [Internet]. [Florianópolis (SC): SES; 2021 [citado em 26 jan. 2021]. Disponível em: http:// www.coronavirus.sc.gov.br/gestao-da-saude/

20. Ministério da Saúde (BR). Doença pelo coronavírus covid-19: Semana epidemiológica 35. Bol Epidemiol [Internet]. 29 ago. 2020 [acesso 26 jul. 2021]. Disponível em: https://antigo.saude. gov.br/images/pdf/2020/September/02/18hBoletim-epidemiologico-COVID-29-final.pdf

21. Cavalcante JR, Cardoso-dos-Santos AC, Bremm JM, Lobo AP, Macário EM, Oliveira WK, et al. COVID-19 in Brazil: evolution of the epidemic up until epidemiological week 20 of 2020. Epidemiol Serv Saude. 2020;29(4):e2020376. doi: https:// doi.org/10.5123/s1679-49742020000400010.

22. Portal Covid-19 Brasil. Monitoramento da taxa de letaldade (óbitos/confirmados) de covid-19: Brasil por estado [Website]. 2021 [acesso 25 jul. 2021]. Disponível em: https://ciis.fmrp.usp.br/covid19/estado-br/

23. Valcarcel B, Avilez JL, Torres-Roman JS, Poterico JA, Bazalar-Palacios J, La La Vecchia C. The effect of early-stage public health policies in the transmission of covid-19 for South American countries. Rev Panam Salud Publica. 2020;44:e148. doi: https://doi.org/10.26633/RPSP.2020.148.

24. Conectas Direitos Humanos. Mapeamento e análises das normas jurídicas de resposta à covid-19 no Brasil. Bol Direitos Pandemia. 14 set. 2020 [acesso 21 jul. 2021];(5):1-18. Disponível em: https://www.conectas. org/publicacao/boletim-direitos-na-pandemia-no-5 
25. Siedner MJ, Harling G, Reynolds Z, Gilbert RF, Haneuse S, Venkataramani AS, et al. Social distancing to slow the US covid-19 epidemic: longitudinal pretest-posttest comparison group study. PLoS Med. 2020 Aug 11;17(8):e1003244. doi: https:// doi.org/10.1371/journal.pmed.1003244.

26. Moraes RFD. Determinants of physical distancing during the covid-19 epidemic in Brazil: effects from mandatory rules, numbers of cases and duration of rules. Cien Saude Colet. 2020;25(9):3393-400. doi: https://doi.org/10.1590/1413-81232020259.21892020.

27. Aith F. Afirmação histórica do direito à saúde $\mathrm{e}$ os desafios para sua efetivação nos estados do século XXI. In: Santos A0, Lopes LT, organizadoras. Reflexões e futuro [Internet]. Brasília, DF: CONASS; 2021 [acesso 20 out. 2021]. (Coleção covid-19; vol. 6). p. 12-27. Disponível em: https://www.conass. org.br/biblioteca/volume-6-reflexoes-e-futuro/

\section{Abstract}

objective: To describe the evolution of decrees and indicators related to COVD-19 in Santa Catarina, Brazil, up until August 2020. Methods: This was an ecological study that analyzed epidemiological indicators and state decrees on social distancing. The decrees were grouped into restriction, maintenance and relaxation. The indicators were mortality, incidence and transmissibility. Results: 179,443 cases and 2,183 deaths were recorded in the period. The incidence rate went from 20.4 cases per 100,000 inhabitants in March to 642.2 in August. Fifteen state decrees were issued. In August, when the bighest mortality rate was observed (13.1 per 100,000 inhabitants), it was found that all decree categories, except one, had been relaxed. Conclusion: The decrees that relaxed social distancing measures were issued early and in disagreement with the epidemiological context in the state.

Keywords: Coronavirus Infections; COVID-19; Mortality; Decrees; Legislation; Epidemiology, Descriptive.
28. Supremo Tribunal Federal (BR). STF reconhece competência concorrente de estados, DF, municípios e União no combate à covid-19 [Internet]. Brasília, DF: STF; 15 abr. 2020 [acesso 20 out. 2021]. Disponível em: https://portal.stf.jus.br/noticias/ verNoticiaDetalhe.asp?idConteudo=441447\&ori $=1$

29. Ferguson NM, Laydon D, Nedjati-Gilani G, Imai $\mathrm{N}$, Ainslie K, Baguelin M, et al. Report 9: impact of non-pharmaceutical interventions (NPIs) to reduce COVID19 mortality and healthcare demand. 2020 Mar 16 [acesso 19 mar 2020]. doi: https://doi.org/10.25561/77482

30. Prem K, Liu Y, Russell TW, Kucharski AJ, Eggo RM, Davies N, et al. The effect of control strategies to reduce social mixing on outcomes of the covid-19 epidemic in Wuhan, China: a modelling study. Lancet Public Health. 2020;5(5):e261-70. doi: https://doi.org/10.1016/S2468-2667(20)30073-6.

\section{Resumen}

Objetivo: Describir la evolución de los decretos e indicadores relacionados con la COVID-19 en Santa Catarina, Brasil, hasta agosto de 2020. Métodos: Estudio ecológico que analizó indicadores epidemiológicos $y$ decretos estatales sobre distanciamiento social. Los decretos se agruparon en: restricción; mantenimiento y flexibilización. Mortalidad, incidencia y transmisibilidad fueron los indicadores. Resultados: Se registraron 179.443 casos y 2.183 defunciones. La tasa de incidencia pasó de 20,4 casos por 100.000 habitantes en marzo a 642,2 en agosto. Se emitieron 15 decretos estatales. En agosto, cuando se observó la tasa de mortalidad más alta (13,1 por 100.000 babitantes), parecía que todas las categorías, con excepción de una, se habian flexibilizado por decretos anteriores Conclusión: Los decretos que flexibilizaron las medidas de distancia social se emitieron precozmente y en desacuerdo con el momento epidemiológico en el estado.

Palabras clave: Infecciones por Coronavirus; COVID-19; Mortalidad; Decretos; Legislación; Epidemiología Descriptiva. 